\title{
Pratiques urbaines et imbroglio spatial : Brazzaville de la fin de la période coloniale au début du XIXe siècle
}

\author{
Jean Félix Yekoka*
}

\section{Résumé}

Née de la volonté coloniale à la fin du XIXe siècle, Brazzaville présente l'image d'une ville artificielle. Son hyper croissance spatiale, qui n'est nullement le résultat d'une quête populaire à optimiser ou à satisfaire le besoin d'habitation, renforce sa fragmentation.

Ville macrocéphale, Brazzaville intègre dans son tissu urbain une gamme d'activités (jeux des propriétaires fonciers, petit commerce, petite agriculture, parkings, lavages auto, maraîchages, marquages, campagnes d'évangélisation, kiosques de téléphonie mobile, etc.) qui prennent d'assaut l'espace public. Il s'affiche à l'intérieur de ces pratiques une dynamique conflictuelle entre différents acteurs en présence, une course effrénée à l'espace public. Ce qui entraîne l'aliénation de celui-ci. Cette aliénation induit une confusion dans l'administration de l'espace public. Au regard des différents enjeux et aux défis auxquels les pouvoirs publics sont confrontés, en matière d'espace public urbain de Brazzaville, des scénarios sont envisageables dans la longue durée.

\begin{abstract}
A product of colonial will at the end of the nineteenth century, Brazzaville presents the image of an artificial city. Its spatial hypergrowth, which in no way results from a popular quest to optimize or satisfy a need for housing, reinforces its fragmentation.

A macrocephalic city, Brazzaville's urban fabric includes a range of activities (real estate, petty trade, small-scale agriculture, parking lots, car washing, market gardening, marking, evangelization campaigns,
\end{abstract}

* IGRAC, Université Marien Ngouabi (Congo). Email : yekokaj@yahoo.fr/ jeanfelixperfection@yahoo.fr 
mobile telephone stands, etc.), which take the public space by storm. Within these practices, the dynamics between the different actors involved is conflictual as they engage in a mad race for public space, which leads to its alienation. The alienation of public space causes confusion in its administration. In response to the different issues and challenges facing the public authorities regarding the urban public space of Brazzaville, scenarios can be envisaged over the long term.

\section{Introduction}

Lorsqu'on s'interroge sur l'espace public urbain de Brazzaville, le diagnostic est vite fait: dans un premier temps, dans la longue durée, l'espace public de Brazzaville est le champ de manipulation et de violence politiques ${ }^{1}$; dans le deuxième temps, tel un jeu de puzzle, cet espace est largement ouvert aux enjeux socioéconomiques incontrôlés. Malgré de multiples réunions tripartites (mairie, police nationale et Ministère de l'administration du territoire) aux fins de réguler le domaine et l'espace publics, ce dernier est toujours ouvert à un ensemble de pratiques (vente des surfaces de terrains par les propriétaires fonciers, petit commerce, kiosques, parkings, cabines téléphoniques, petite agriculture urbaine, etc.) qui défient l'administration publique en la matière.

En effet, la « consommation » quotidienne de l'espace public par ses usagers, dans un contexte urbain, rassemble de nombreux facteurs conduisant à un changement social en latence et, sans doute, à une croissance spatiale rapide incontrôlée. Mais à côté de cette croissance spatiale urbaine, il ne faut pas perdre de vue la croissance démographique qui fait de Brazzaville une ville macrocéphale.

Estimée à 122000 habitants en 1961 et à 420000 habitants en $1981^{2}$, Brazzaville compte, selon les estimations actuelles, plus de 900000 habitants. L'augmentation démographique, qui a une incidence sur les conditions de vie urbaine, pose de vrais problèmes d'administration de l'espace public. Les enquêtes menées à Brazzaville montrent que 21,83 pour cent seulement des occupants de l'espace public reconnaissent aux autorités publiques un pouvoir régalien sur l'espace urbain ; 57,33 pour cent ignorent tout pouvoir aux autorités publiques en matière de gestion de l'espace urbain. Parallèlement, environ quatorze pour cent reconnaissent avoir reçu l'autorisation des pouvoirs municipaux pour occuper une surface publique à des fins diverses. Formellement, environ 6 pour cent des utilisateurs d'espace public sont détenteurs d'un permis d'occuper.

Au regard de ces statistiques, tout porte à croire qu'il est difficile d'établir une péréquation entre le contrôle efficient de l'espace public, la densité des pratiques qui s’y opèrent et le rôle des autorités administratives à réguler l'espace public d'une ville en croissance incontrôlée. Devant la 
méconnaissance des pouvoirs publics sur l'espace public qui tend à s'optimiser, à côté des faiblesses ou de l'insuffisance des capacités institutionnelles qui jettent le flou sur les questions d'espace public à Brazzaville, cette contribution vise une série de prolégomènes qui nourrissent la confusion entre actions urbaines et politique administrative en matière de gestion de l'espace public. La dialectique entre pratiques urbaines et espace public permettra de vérifier également l'existence de conflits d'intérêts au sens de Jean Bauchard (1981).

Quatre points essentiels constituent la charpente de cette étude. Le premier tente de faire une mise au point de l'espace public en rapport avec les questions irrédentistes à Brazzaville. Il s'agira de montrer que le premier trait de confusion spatiale résulte de la coexistence difficile entre droit coutumier et droit moderne. L’imbroglio spatial à Brazzaville mérite, pour sa compréhension, une typologie, un classement des différentes opérations publiques qui se font quotidiennement sur l'espace public. C'est le deuxième point de cette réflexion. Dans la troisième approximation, cette contribution souligne les dessous administratifs dans la gestion de l'espace public urbain. Le dernier point tente d'envisager des scénarios de l'espace public à l'horizon 2025.

\section{Actions et omniprésence des propriétaires fonciers sur la scène de l'espace urbain}

L’espace sur lequel Brazzaville évolue appartient aux Teke. Leurs toponymes, qui ont progressivement intégré le tissu urbain, ont fini par confirmer la preuve linguistique et historique à cet effet. En tant que propriétaires fonciers et au nom du primat ontologique qui les unit à la terre, ces derniers ont depuis toujours influencé, interagi, voire compliqué l'écheveau de l'organisation de l'espace urbain de Brazzaville.

En effet, dans la période qui a suivi les indépendances, le développement urbain a pris une ampleur considérable, conduisant les pouvoirs publics à mettre en œuvre, à partir de la deuxième moitié des années 70, de nombreuses et multiples formes de moyens visant l'orientation et la maîtrise de l'espace urbain. Malgré les manifestations des pouvoirs publics, certes très limitées, l'espace public s'est et a toujours été caractérisé par un assujettissement à grande échelle des prérogatives de l'Etat à des actions privées des chefs de terres et autres propriétaires fonciers qui agissent pour leur compte sur les périmètres urbains ${ }^{3}$ et, surtout, sur les espaces dits publics.

Il convient de dire que malgré l'initiation dynamique des textes sur la réalisation de l'urbanisation et le contrôle plus ou moins efficace des domaines et espaces publics par l'administration publique, l'omniprésence et 
l'omnipotence des propriétaires fonciers sur les différentes scènes de gestion et de contrôle de l'espace public se signalent avec vitalité. On note d'ailleurs leurs actions dans le lotissement de la ville à partir des années qui ont suivi l'indépendance. D'importantes surfaces nues sont loties par ces chefs traditionnels aux abords de Brazzaville. Avec les nécessités économiques qui tendent à satisfaire les demandes sociales, notamment dans l'acquisition d'un terrain bon marché en vue de construire un logement personnel, avec ou sans la complicité des agents de la mairie, les propriétaires fonciers vendent anarchiquement des terrains. Ces ventes se font sans le moindre souci d'assainissement, de salubrité et de protection des surfaces réservées pour une utilité publique. Dans cette perspective, on attribue volontiers à ces propriétaires la responsabilité de limiter l'importance numérique et spatiale des espaces publics.

Toutefois, il faut noter la permanence des conflits entre ces propriétaires et les pouvoirs publics. En analysant avec minutie ces conflits (Ziavoula 1988:26), on note qu'à chaque fois que l'administration municipale ne dispose pas d'espace pouvant faire l'objet d'un lotissement à l'intérieur de sa zone de compétence, elle procède à l'expropriation. Outre l'expropriation, elle déclare le retour au domaine des terrains déjà attribués et dont la mise en valeur est insuffisante, suivant les critères définis par l'administration. Seulement, l'arrêté d'expropriation ou du retour au domaine n'empêche pas les propriétaires fonciers de continuer à vendre les espaces publics inclus dans les surfaces de terrains qui relèvent de leur propriété. L'administration, par le biais de la Direction de la gestion foncière urbaine et de recherche (DIGEFUR), se heurte ainsi continuellement à de nouveaux logiciels fonciers dans la gestion et l'administration des espaces publics.

A l'analyse, le regain de tension entre pouvoir public et propriétaires fonciers est à mettre à l'actif des contradictions issues des Constitutions de 1963 et 1969, et des résultats de la Conférence nationale souveraine de 1991. En 1963, en effet, la terre fut nationalisée sur toute l'étendue du territoire national. S'inspirant de l'administration coloniale, en matière de gestion des espaces et domaines publics, le pouvoir d'antan avait mis en mouvement toute une batterie de textes administratifs, une façon pour lui, certes, d'avoir une mainmise effective sur la totalité des espaces issus du territoire national. En 1969, la terre est déclarée "propriété du peuple » avec la célèbre formule « tout pour le peuple, rien que pour le peuple ». A cette époque, l'espace public est désigné sous l'expression de «domaine populaire ». Dans la rhétorique marxiste, le concept " populaire » est très caractéristique. Non seulement il est connoté, mais il est aussi fortement chargé de pouvoir extraordinaire, en ce sens qu'il a permis d'intéresser le maximum d'individus possible à s'approprier l'espace ${ }^{4}$. Dès lors, une course 
effrénée à l'espace public s'est engagée à Brazzaville par différentes catégories de classes sociales : fonctionnaires de l'Etat et du secteur privé, commerçants, étrangers, etc.

En marge de la Constitution de 1969, la loi N 52/83 du 21 avril 1983 portant code domanial et foncier en République Populaire du Congo « avalait» le pouvoir et l'action populaires sur la terre. Dans son article $1^{\text {er }}$, cette loi déclare que «la terre est, sur toute l'étendue du territoire de la République Populaire du Congo, la propriété du peuple représenté par l’Etat ». De facto, l'Etat récupérait ainsi toutes les surfaces de terre établie sur son territoire. Mais avec la Conférence nationale souveraine de 1991, l'Etat reconnaissait le droit privé de la terre, donc de plusieurs surfaces vides considérées jusque-là comme espaces publics. Ces surfaces allaient donc rentrer dans le circuit dynamique des opérations de vente des espaces. Devant les réseaux fonciers qui naissent et qui se structurent aux rixes avec l'administration, « les autorités municipales et nationales se retrouvent dans l'impasse, en essayant de démêler différentes formes logiques et historiques de documentation, pour réunir les transactions foncières réalisables » (Maliq 1998:43).

Il ressort de l'analyse des rapports antagoniques entre propriétaires fonciers et pouvoirs publics que deux types de régimes fonciers ont cohabité au Congo, depuis la période coloniale jusqu'à maintenant. Le premier est évidemment le plus ancien, le droit coutumier dont on connaît les caractéristiques essentielles : la terre appartient au groupe clanique dont elle assure l'existence par la cueillette, la chasse, la pêche, l'agriculture, etc. Les membres de ce groupe ont, à l'intérieur de ce "domaine », un droit d'usage permanent selon les règles traditionnelles. Le deuxième est celui introduit par l'administration coloniale en 1899. C'est un régime du Code civil qui fait de l'Etat le propriétaire éminent de toutes les terres (Vennetier 1985:199), donc de tout espace dit public. Le premier régime est immuable, le deuxième ne l'est point. Son instabilité est due aux pressions et enjeux sociaux exercés quotidiennement sur la terre et sur l'espace public. Ces pressions poussent les pouvoirs publics à revisiter maintes fois le Code civil foncier. La dernière révision date de décembre 2008. Contre toute attente, cette dualité du régime juridique de la terre favorise l'accès d'un plus grand nombre d'individus à l'espace public.

Parallèlement aux options contradictoires entre pouvoirs publics et maîtres traditionnels de la terre urbaine, l'enjeu majeur de la course à l'espace a fini par concrétiser, voire achever la fragmentation spatiale déjà perceptible pendant la période coloniale. Cette fragmentation, qui s'est accompagnée de la ségrégation ethnique, régionale et identitaire, a fortement polarisé l'espace urbain en Brazzaville nord, où habitent majoritairement les populations 
originaires du Nord Congo et en Brazzaville sud, habité par des groupes ethniques issus des Départements sud du Congo. Mais le vrai problème de cette fragmentation est sans doute la restriction du pouvoir des autorités municipales à administrer pleinement les espaces publics ${ }^{5}$ sous leurs compétences. On peut même penser que les espaces publics établis en banlieue échappent systématiquement, sinon presque, au contrôle municipal, puisque même les espaces situés au cœur de la ville sont victimes de sollicitation illicite et de pratiques urbaines à grande échelle.

\section{Les pratiques urbaines dans les espaces du centre de Brazzaville}

Nous avons vu que l'espace des quartiers périphériques de Brazzaville est continuellement victime des opérations marchandes pour lesquelles leurs acteurs mettent au pilori la DIGEFUR. Mais si le thermomètre des espaces périphériques donne des résultats inquiétants en matière d'administration spatiale, il n'en demeure pas moins que les espaces des quartiers situés au cœur de Brazzaville (Moungali, Poto-Poto, Ouenzé, Plateau des 15 ans, Bacongo, Makélékélé, Talangaï, centre-ville, etc.) soient eux aussi abusés, aliénés. Les différentes pratiques qui s’y opèrent méritent, pour leur compréhension plus ou moins effective, un classement, une typologie, une catégorisation, une hiérarchisation. Car, ces pratiques déterminent la connaissance effective d'espace urbain plus ou moins étendu, cette connaissance, à son tour, influence et explique en partie les images citadines.

\section{Les parkings, les lavages auto et les conteneurs}

Une simple promenade à l'intérieur des quartiers précédemment énumérés permet de se rendre à l'évidence que l'espace public est cloisonné, saturé par les parkings, les lavages auto et les conteneurs. Dans les années 1960 et 1970, la mairie de Brazzaville avait prévu un parking automobile, mais avec le développement de la ville et du transport dans le secteur privé ${ }^{6}$, ce parking a vite été abandonné, parce qu'incapable d'accueillir un nombre important de voitures.

Afin de régler l'épineux problème lié au parking, les pouvoirs publics avaient demandé, dans une circulaire $\mathrm{n}^{\circ} 127$ du 21 mai 1969, aux usagers d'espace public d'obtenir l'autorisation de l'autorité compétente. Après examen de la demande, l'autorité délivre un alignement indiquant les limites de la voie publique au droit de l'espace du parking. Cette exigence est valable pour les lavages auto et pour les conteneurs. En 2004, l'autorisation d'occuper un espace public en vue de l'installation d'une station de lavage automobile est taxée à 3000F/m linéaire le mois ${ }^{7}$. Mais l'analyse des textes administratifs initiés sans fin dans la gestion du domaine et de l'espace publics prouve que 
peu d'individus obtiennent effectivement l'autorisation d'occuper un espace jadis réservé au public. Selon les résultats de nos enquêtes, environ 81,9 pour cent des Congolais et/ou d'entreprises occupent illicitement l'espace public. Les parties du sol aménagées en parkings, lavages auto ou en conteneurs contrevention de la voirie.

Il n’est pas rare de lire des plaques portant la mention « Parking réservé » ou « Parking privé » sur une partie soit de terrain, soit du trottoir attenant à la voie publique. La mention « Parking réservé » est très parlante et peut signifier bien des choses. Elle peut, par exemple, symboliser la présence d'une force qui rivalise ou qui méconnaît tout simplement le pouvoir de la Mairie sur l'espace urbain. Parler de "force » signifie indexer certaines " personnes intouchables » qui, bénéficiant des pouvoirs et de l'influence de certaines autorités de l'Etat, contournent les procédures administratives d'acquisition d'espaces.

Comme nous le verrons plus loin, la présence illicite des conteneurs, lavages auto et parkings dans les quartiers de Brazzaville est consécutive au dysfonctionnement administratif, notamment entre la mairie centrale, les arrondissements, les chefs des quartiers et la force publique. Par exemple, certains maires des arrondissements autorisent, sans passer par les services de la Direction de la propriété de la Ville (DPVB) et la DIGEFUR, à certaines personnes d'installer un conteneur, un lavage auto ou un parking sur un espace public ${ }^{8}$. La DPVB est l'institution administrative chargée du contrôle sur le terrain de la régularité ou au non-paiement des redevances afférentes. Mais si les parkings et les conteneurs sont omniprésents sur les espaces publics, leur visibilité est bien moins apparente que celle d'autres artisans qui occupent les mêmes espaces pour des intérêts économiques liés au petit commerce et aux petits métiers.

\section{Le petit commerce et les petits métiers}

L'amélioration de l'ordinaire de certains ménages passe par la pratique des petites activités économiques. Le marché en tant que cadre approprié de mise en exergue des opérations de vente et d'achat ne suffit pas pour tous les citadins. Ainsi, certaines couches sociales choisissent l'espace public comme cadre propice de liquidation et d'écoulement et/ou d'acquisition des produits commerciaux.

Entre autres activités commerciales qui se réalisent dans les espaces publics, citons le commerce des habits, la vente des produits vivriers (arachides, banane, manioc, yaourt, gingembre, papaye, etc.), les produits combustibles (pétrole, gasoil, huile moteur, etc.), les matériaux de construction (gravier, planche, sable, etc.), etc. 
A côté de cette quincaillerie de produits viennent se juxtaposer les cabines téléphoniques et les librairies par terre. En effet, depuis la fin du cycle de violence qui a secoué le Congo entre 1997 et 2002, les cabines téléphoniques et les librairies par terre se sont insérées progressivement dans la carte des pratiques commerciales qui se font dans des espaces destinés au public. Ces cabines et librairies ont pris d'assaut les trottoirs et les arrêts de bus où elles rivalisent désormais d'égal à égal avec les femmes vendeuses des produits de consommation directe.

En marge des activités commerciales déjà mentionnées, se pratiquent d'autres activités d'une autre dimension économique. Ces activités, qui relèvent du monde informel, sont principalement la bijouterie, la peinture, la sculpture, la vannerie, la coiffure, la décoration. Ces activités, qui sont essentiellement masculines, se pratiquent parfois dans des parties terminales de certaines administrations publiques sans aucune autorisation écrite dûment acquise des autorités de ces administrations. Tout suit le circuit informel, celui des pots de vins et d'autres relations interpersonnelles.

Environ 53,11 pour cent des personnes exerçant le petit commerce contre 39,4 pour cent de ceux exerçant des petits métiers ignorent que toute installation sur un espace public est subordonnée à l'acquisition préalable d'une autorisation administrative. Pour ces marchands obnubilés par la soif du gain, le bon positionnement vaut mieux que tout. En revanche, ces acteurs subissent paradoxalement, mais de façon discontinue, les affres des opérations de déguerpissement organisées par la mairie, conjointement avec la force publique. Interrogés à leur sujet, nombre d'agents municipaux pensent que cette catégorie d'occupants d'espace public cause plus de problèmes à la mairie, dans la mesure où beaucoup d'entre eux pensent que lorsqu'un espace est vide, il n'appartient à personne; il échappe au contrôle de qui que se soit, même à celui des pouvoirs publics.

En réalité, de telles déclarations tentent de camoufler les faiblesses de la mairie et d'autres secteurs administratifs congolais dans la vulgarisation effective des textes souvent initiés. C'est plutôt un camouflet qui ne ramène point les choses à l'horizon-monde, car comment comprendre que certains espaces et arrêts de bus soient le monopole privé de quelques hommes politiques à l'intérieur des quartiers qu'ils contrôlent ? Prenant le spectre de leur statut politique, ces individus initient une administration parallèle à celles légalement connues (celle de la mairie et de la police nationale), aux fins de collecter les taxes de chargement de bus, d'occupation d'espace public et/ ou de salubrité publique. Eclectique des pratiques urbaines sur l'espace public, éclectique administratif, politique et, certainement, des petites activités agricoles sur l'espace public urbain. 


\section{Les activités agricoles dans quelques espaces publics}

Il s'agit des activités qui se pratiquent sur des espaces qui arborent les bâtiments publics, les espaces marginalisés par l'Etat et celles concernant la ceinture maraîchère de Brazzaville. Ces activités peuvent être classées en deux catégories différentes :

\section{La petite agriculture urbaine}

Elle sert à l'autoconsommation, avec, toutefois, une faible quantité vouée à la commercialisation. En essayant de mettre en perspective ces activités avec l'administration publique en matière de gestion spatiale, il est clairement établi que les pouvoirs publics laissent travailler les femmes dans les espaces signalés pour des raisons de salubrité publique. Ces femmes qui ne sont inquiétées ni par la mairie, ni par la police nationale, comme le sont parfois leurs homologues qui sont sur les terrasses, jouent le rôle d'auxiliaire de propreté de la mairie. Mais le vrai problème avec elles est qu'elles ont tendance à privatiser les espaces qu'elles cultivent souvent. Chaque année, elles sont présentes sur les surfaces dont elles estiment être les " propriétaires ». Les stratégies des autorités publiques de les laisser gérer seules ces espaces soulignent en rouge leurs faiblesses en matière de gestion et d'administration des espaces publics. Cette démarche non savante peu générer, au fil des temps, des conflits entre les descendants de ces femmes et le pouvoir public.

\section{Les cultures maraîchères et leur mise en quarantaine}

Les activités liées à la ceinture maraîchère de Brazzaville sont essentiellement marchandes, dans la mesure où c'est cette ceinture maraîchère, par le biais des jardiniers, qui alimente les marchés de Brazzaville en légumes. Mais tout comme les espaces qui alourdissent le climat entre l'administration publique et les propriétaires fonciers, la ceinture maraîchère ravive le syndrome de la violence spatiale ${ }^{9}$. Dans les quartiers nord de Brazzaville, l'espace met souvent en confrontation quatre forces: les pouvoirs publics, les acheteurs de terrains, les propriétaires fonciers, les jardiniers. Ces derniers sont réduits en minorité par les propriétaires fonciers. Sollicitant les services des avocats dans les revendications des terrains, la rixe s'anime, dans une dimension maximaliste, entre pouvoirs publics et propriétaires fonciers ${ }^{10}$. Dans le but d'arrêter le recul spatial observé dans le périmètre urbain, depuis plus de deux décennies, les autorités municipales et ministérielles instituent des mesures conservatoires de la ceinture maraîchère. Malheureusement, les mêmes autorités délivrent des titres et permis fonciers à des acheteurs de terrain dans cet espace réservé pour des utilités publiques ${ }^{11}$. Dans la confusion administrative, il n'est pas rare de trouver deux acheteurs d'un même espace, avec chacun un permis d'occuper légalement produit par les services officiels de la Mairie. 
Aujourd'hui plus que hier, l'espace est menacé de disparition, notamment avec l'entrée en scène et dans la course à l'espace public par les « Nouveaux riches » et les « Karachikas ». Les Nouveaux riches et les Karachikas sont deux termes linguistiques urbains qui sont nés à Brazzaville au lendemain de la guerre du 5 juin 1997. Ils concernent les gens qui se sont brusquement enrichis par le pillage et les pratiques magiques consistant, en vue de s'enrichir, à vendre une partie de leur corps. Ces Karachikas s'habillent et construisent leurs maisons selon le modèle nigérian, notamment celui véhiculé dans et par les films nigérians. Ces films Karachikas sont largement suivis à Brazzaville, via Kinshasa, où les chaînes de télévisions privées sont en exponentielle croissance numérique. Ces médias diffusent ces films sans répit. Devenus acteurs dynamiques dans les pressions spatiales qui s'exercent dans la ceinture maraîchère, entre la municipalité et les propriétaires fonciers, ces nouveaux riches et Karachikas participent à jeter le flou et la confusion dans l'administration spatiale urbaine par la voie de la matrice efficace de la corruption et la concussion. A l'heure de la mondialisation, les Karachikas ${ }^{12}$ et les Nouveaux riches impriment à la ville, tant soit peu, la vitrine d'une ville artificielle certes, mais en quête de modernité, notamment avec « la municipalisation accélérée ».

\section{Les sociétés privées de construction routière à l'heure de la municipalisation accélérée}

La «municipalisation accélérée » est un axe de la Nouvelle Espérance ${ }^{13}$. Définie comme stratégie de développement et d'urbanisation rapide d'une ville pendant une période bien déterminée, la municipalisation accélérée consiste à canaliser plusieurs projets et une part significative du budget d'investissement annuel sur une ville dûment choisie par le président de la République, lors de son message (discours) à l'Assemblée, où il fait le bilan de l'année. Cette municipalisation concerne en premier lieu les chefs-lieux des départements, y compris leurs agglomérations satellites. Etant tournante, la municipalisation accélérée permet au gouvernement d'émailler l'ensemble du pays en infrastructures de base et d'atteindre ainsi plusieurs objectifs en un temps record.

Brazzaville emboîte le pas de Pointe Noire, Impfondo, Dolisie et Owando. Deux ans (2008 et 2009) durant, quelques entreprises (SGE-C Congo, SOCOFRAN, ESCOM, etc.) ont été retenues par le gouvernement pour réaliser les projets retenus ${ }^{14}$. Ces entreprises, qui avaient signé des contrats avec le gouvernement et la Direction générale des grands travaux, ne s'étaient pas réellement intéressées à la Mairie et à la préfecture qui sont, sur le plan administratif, les "maîtresses » de Brazzaville. Sans le mandat de la municipalité, dans l'exécution des travaux de ladite municipalisation accélérée, 
ces entreprises utilisent à leur manière les espaces publics. Ceux-ci servent de lieux de stockage de sables, briques, graviers, etc., empêchant ainsi la libre circulation des personnes et des biens. Or, ne relevant point du Ministère des travaux publics, ces entreprises privées devraient se plier aux normes administratives municipales en matière de gestion et d'administration de l'espace public urbain.

\section{De l'espace public enchanté à l'espace public déchanté}

L'idée de l'enchantement et du « déchantement » de l'espace public vient de ce que durant l'ère coloniale et pendant la post colonie, l'espace public a fait l'objet d'une administration efficace, avec des textes devant régulant toutes les formes de contacts entre les citoyens et l'espace lui-même. L'espace public - surtout le jardin public - était sacré. Cette sacralité tenait au fait que pour réussir à imprimer le schéma urbain dans la mémoire (l'imaginaire) collective des citadins, il fallait donner à l'espace le caractère inviolable. C'est ainsi qu'à chaque type d'opérations à réaliser sur l'espace public correspondait et correspond encore un type de droit à payer en amont ; et à chaque type de violation de l'espace correspondait et correspond encore un type de sanctions ${ }^{15}$ et/ou d'amende.

La période coloniale est passée. Au nom de la modernité, la ville intègre progressivement en son sein des réalités culturelles nouvelles qui suscitent parfois l'étonnement et la surprise de certains habitants. En rapport avec la vie urbaine, l'espace public est ouvert à des pratiques culturelles obscènes qui leur déchantent. L'exhibition du nu, des danses et chansons obscènes sur la place publique lors des cérémonies funéraires se fait en marge des normes administratives en matière de gouvernance urbaine. En d'autres termes, le nu tel qu'il est exposé au public dans des milieux non autorisés porte atteinte à l'ordre public et à l'autorité de l'Etat. L'occupation anarchique des espaces publics, des portions de rue, pour des besoins funéraires se fait en parallèle avec les pratiques religieuses sur l'espace public ${ }^{16}$.

L'observation de l'effervescence religieuse à Brazzaville " sécrète " d'autres mécanismes évangéliques. En effet, contrairement aux églises traditionnelles (catholiques, salutistes, protestantes, kimbanguistes) qui bâtissaient dans des espaces éloignés de la ville, les églises - celles dites Assemblées de réveil ou Eglises pentecôtistes - actuelles s'insèrent plutôt au cœur du tissu urbain où elles prennent d'assaut l'espace public comme lieu d'évangélisation des âmes. Mais la course aux âmes par la tenue des campagnes d'évangélisation dans des espaces publics n'est plus le monopole des seules assemblées chrétiennes, elle intéresse dorénavant les églises catholiques et protestantes. Hier terrées dans leurs cathédrales, ces églises ont fini par s'intéresser à la rue dans l'exercice de leurs pratiques théologiques. 
L'espace public de Brazzaville s'ouvre largement au public le plus démuni sous diverses formes ambulantes, sédentaires et permanentes. Outre le rôle désormais classique de négociation d'une sécurité vitale de la majorité de la population blessée par les barbelés de la pauvreté qu'il joue, l'espace public semble devenir assez trop le lieu des échanges et des débats politiques. Une sorte d'hémicycle symbolique populaire qui se signale et se particularise par l'absence de chefs couronnés, investis à qui reviendrait l'ultime devoir de diriger les débats. Dans le contexte politique, les débats sur la place publique se polarisent très souvent autour des velléités démocratiques, notamment celles des enjeux électoraux. Ce phénomène s'observe depuis les élections de 1958, 1992 et 2002. Aujourd'hui, les rassemblements populaires sur l'espace public se polarisent autour des élections présidentielles de 2009 qui pointent à l'horizon. Au sein de cette structure informe et informelle, les populations tentent de déposséder les élus (députés) nationaux de leurs prérogatives, afin d'exprimer elles-mêmes à qui veut les entendre ce qu'elles attendent des pouvoirs publics. Tel un jeu de puzzle, les discours populaires caricaturent l'Etat. Ainsi, l'espace public est devenu le fer de lance de plusieurs pratiques urbaines. Il est à la croisée des chemins entre quête de reconnaissance et de leadership, entre besoins économiques et violences urbaines, entre pratiques culturelles désacralisantes et conquête des âmes. Tout cela est révélateur des transformations d'une société urbaine en transition démographique.

\section{Parcellisation de l'espace public urbain}

Les différentes pratiques exercées sur l'espace public urbain créent et induisent des référents d'identification et de séparation de pouvoir des périmètres occupés. Cette parcellisation de l'espace public par ses usagers se matérialise par des symboles sociaux dont le marquage et la personnalisation en sont les expressions les plus connues et les plus répandues.

Le marquage est une manière de signer ${ }^{17}$ un espace par des inscriptions rendues identifiables par l'action de l'utilisateur. Ce terme englobe l'ensemble des objets utilisés à cet effet, car ils sont en quelque sorte identifiés par l'action. Féindangai (2004:244), qui cite Goffman, distingue trois types de marqueurs :

- les marqueurs centraux qui sont placés au centre d'un territoire et en établissent la possession ;

- les marqueurs-frontières qui indiquent la ligne de séparation entre deux territoires ;

- les marqueurs signets qui inscrivent la marque personnelle sur un objet ou dans un espace. 
Ces différentes formes de marqueurs sont très polysémiques et très caractéristiques à Brazzaville où les « prédateurs » de l'espace les associent volontiers aux noms qu'ils attribuent à leurs « entreprises » (kiosques, salons de coiffure, cabines téléphoniques, etc.) et aux produits qu'ils commercialisent. Tout cela élabore une anthropologie linguistique et religieuse des temps modernes filant en ligne droite : « Dieu m’a béni », « Dieu merci phone ", « Coin du Christ », « Quartier général », « Grâce sublime », « Dieu est amour », «La cour des grands », " Grâce phone », etc. Ces différents noms attribués aux espaces occupés et aux types de pratiques qui s’y opèrent sont à inscrire dans une perspective publicitaire qui traduit à la fois une course au clientélisme, une ascension spirituelle symbolisée par des bénédictions divines sublimées et dévoyées, et une rupture avec la tradition des années 80 où les noms des kiosques, boutiques, salons de coiffure, etc. exaltaient et faisaient rêver l'Europe.

La parcellisation de l'espace public n'épargne pas l'imaginaire collectif. Elle insère en son sein les segments de pouvoir individuel sur l'espace. Ainsi, la dénomination individuelle de l'espace rendu progressivement privé contribue à la création d'un ordre symbolique, c'est-à-dire à la mise en exergue d'une logique cohérente et harmonieuse qui cherche la création d'un lien inséparable entre l'espace public occupé, son occupant et tous ceux (famille, amis, collègues, etc.) qui lui sont unis. C'est donc une appropriation de l'espace qui se veut pérenne, d'autant plus que l'espace spolié est assimilé à un patrimoine clanique qui se transmet aux générations futures aussi longtemps que possible.

\section{Les dysfonctionnements administratifs dans la gestion de l'espace public urbain}

Les différents acteurs sociaux qui agissent sur l'espace public de Brazzaville permettent, à cause de la confusion qu'on observe sur le terrain, que soit analysé avec minutie le circuit administratif en matière de gestion de l'espace public. Mais on peut souligner très tôt les différents dysfonctionnements qui émaillent ce circuit, cette politique spatiale des pouvoirs publics en vigueur à Brazzaville. Relevant en plus que, selon l'administration congolaise, l'espace public fait partie du domaine public. En tant que tel, l'espace public ne dispose pas, à notre connaissance et selon nos investigations, d'une politique administrative spéciale à laquelle on peut se référer.

Et en matière d'administration du domaine et de l'espace publics, plusieurs structures administratives agissent, et ce, sans respecter les étapes de la collaboration. Entre autres structures, il faut citer la mairie centrale, par le biais de la DIGEFUR et la Direction des travaux municipaux (DTM), les 
arrondissements, le Ministère de l'urbanisme, par le truchement de ses délégués en poste dans tous les arrondissements.

Le processus légal d'établissement ou d'occupation de l'espace public est le suivant : les clients et les contribuables introduisent à la mairie centrale une demande dans laquelle ils expriment, en justifiant, le désir d'occuper une partie de l'espace public et/ou du domaine public en lieu et place de leur choix. La demande est ensuite envoyée à la DIGEFUR pour examen et pour des formalités administratives. La DIGEFUR instruit à cet effet une enquête technique sur le terrain. Les besoins de l'enquête consistent à se rassurer si la demande des clients ne troublera point l'ordre et la sécurité publics, si elle garantit les problèmes urbains. Pour ce faire, la DIGEFUR recourt au service de la police nationale et au chef de quartier où l'espace est sollicité. Si le dossier est conforme, la DIGEFUR dresse un ordre de profit de la commune pour paiement des taxes fixées ${ }^{18}$ par la mairie: cinq cent francs par mètre carré et par trimestre tout le temps de l'occupation (Ayessa 1999:54). Mais le manque de suivi de cette taxe par la DIGEFUR fait qu'elle prend le contenu de l'impôt indirect. Après un ordre de perception qui ne dure qu'un trimestre, en effet, on demande au contribuable d'aller verser lui-même l'argent aux dates prévues.

Administrativement, les autorisations sont établies en trois exemplaires reparties comme suit : le primata est remis au contribuable, le duplicata reste à la DIGEFUR, le trimata est envoyé à la Direction des finances, au service de la recette. Mais la DIGEFUR ne dispose ni d'un répertoire spécifique, ni d'un registre, ni d'une grébiche pour les dossiers liés à l'espace public, ce qui fait que certains dossiers ne sont pas transmis au service de la recette. C'est un des maillons de dysfonctionnements administratifs de l'espace public. Mais toutes ces démarches administratives ne sont suivies que par un nombre réduit d'individus (14\%, selon les résultats de nos enquêtes).

En outre, la loi 7-95 du 21 mars 1995 portant organisation et fonctionnement des collectivités locales de moyen exercice alimente les incohérences administratives, notamment entre la mairie centrale et les arrondissements. En effet, selon les articles 71 et 75 de cette loi, « le Conseil d'arrondissement délibère sur toutes les matières en sa compétence (...) : la lutte contre les installations et les constructions anarchiques sur les trottoirs et les places publiques », de plus « le Conseil d'arrondissement donne son avis sur (...) les autorisations et l'utilisation du sol dans les arrondissements délivrés par le maire de la commune.» Etant investis d'un tel pouvoir (Article 71), dans la pratique, les maires des arrondissements ne consultent pas le maire communal, ils délivrent, sans son accord, les autorisations d'occupation 
du domaine et de l'espace publics. Les maires des arrondissements sont donc omnipotents dans la délivrance des permis d'occupation de l'espace public ${ }^{19}$. Pour le faire, ils ne consultent pas le maire de la commune comme le leur recommande la loi, mais plutôt les délégués au Ministère de l'urbanisme, qui travaillent dans leurs structures. Ces délégués qui ne dépendent pas de la mairie centrale, passent outre les avis de la DIGEFUR et de DTM dans les autorisations qu'ils préparent à la signature des maires d'arrondissements (Ayessa 1999:58), ce qui fait que les tarifs qu'ils proposent ne sont pas ceux dûment arrêtés par la DIGEFUR. Parce que dépositaires du pouvoir de l'Etat dans les arrondissements soumis à leur administration et parce qu'ils se soustraient volontairement de la mairie centrale de qui ils sont le prolongement, les maires des arrondissements amplifient la confusion qui règne sur l'espace public de Brazzaville.

Par ailleurs, il est courant d'observer la pagaille qu'organisent ces délégués au Ministère de l'urbanisme, notamment avec la construction sur des collecteurs, sur des lieux de stationnement, sur les caniveaux, sur les lieux publics, etc. En délivrant les permis d'occupation des lieux publics, les maires des arrondissements ne prennent pas le soin de transmettre une ampliation à la DIGEFUR et à la DTM pour régularisation, au service de la recette pour des besoins de contrôle administratif. Le paradoxe est que dans les arrondissements, il n'existe pas de répertoires sur l'espace public, voire sur le domaine public. Malheureusement, quand surviennent les contentieux, les plaignants (les occupants de l'espace public) se présentent à la mairie centrale pour chercher à faire valoir leur droit, alors qu'au départ les services habiletés de la mairie centrale n'étaient pas informés de la procédure d'occupation de l'espace, ce qui fait d'ailleurs qu'il y a beaucoup de victimes des opérations de déguerpissements que la mairie centrale lance en partenariat avec la police en direction des occupants illégaux d'espace public. Mais ces déguerpissements constituent eux-mêmes un véritable casse-tête dans la mesure où certains occupants sont détenteurs des titres délivrés par certains maires d'arrondissements ${ }^{20}$. Les mêmes causes produisant les mêmes effets, dans cette atmosphère chrysogène, certains espaces publics libérés grâce aux opérations de déguerpissements ont été transformés en poubelle publique, avec tout ce qu'elle engendre comme problème de pollution.

En parallèle de la DIGEFUR qui est le service autorisé à délivrer les autorisations d'occupation des domaines et espaces publics, des maires des arrondissements qui s'arrogent le pouvoir d'aliéner l'espace public en octroyant des titres et permis d'occuper des surfaces aux demandeurs d'espace, des délégués à l'urbanisme, les chefs des quartiers aussi se permettent de délivrer les permis d'occuper aux gens dans les quartiers 
qu'ils contrôlent. Seulement, ces permis ne sont pas écrits, ils sont constitués du seul statut de chef de quartier qu'ils ont. Les graves dysfonctionnements qui émaillent les différentes étapes de délivrance d'autorisation d'occupation de l'espace public entraînent avec eux la non maîtrise de gestion et de recouvrement des taxes municipales issues de l'espace public. Ces dysfonctionnements sont d'autant plus graves qu'il existe plusieurs centres ${ }^{21}$ de délivrance des titres ou permis d'occuper de l'espace public et/ ou du domaine public. Déjà, pour le seul secteur du domaine public dans lequel l'espace public s'insère, il y a trop de structures à la mairie centrale (DIGEFUR, DAG, DTM, DFM et la Communication), ce qui pose d'énormes problèmes de gestion des données, de définition et de séparation de pouvoir.

Il s'est donc établi une confusion au sein de l'appareil municipal qui bloque l'appareil administratif en matière d'administration spatiale. Cette confusion participe à stériliser l'espace public de plus en plus abandonné aux pratiques urbaines. La logique administrative est systématiquement remplacée par la volonté de certaines autorités municipales de coopérer directement avec les demandeurs d'espace. De ce fait, l'espace public de Brazzaville est à l'épreuve de la fiction normative. Il faut donc une logique fonctionnelle qui puisse sortir impérativement les textes administratifs de cette fiction, de leur immobilisme.

En outre, il sied de souligner que l'espace public est un secteur qui intéresse différents départements (mairie, police, préfecture, etc.) administratifs. Et chaque secteur agit presque sans tenir compte du pouvoir que les autres ont sur l'espace public. L'impression qui se dégage est celle de l'inexistence d'une feuille de route, d'un schéma directeur sur l'espace public autour duquel chacune des parties se reconnaît. Ainsi, la qualité des rapports entre les différentes structures de l'administration municipale et les services départementaux de la police nationale devrait être définie ou redéfinie et harmonisée, notamment avec la création de la préfecture de Brazzaville. Il s'agit des rapports entre le directeur départemental de la police nationale et le préfet de la ville de Brazzaville ; le directeur départemental de la police nationale, commissaire central de la ville de Brazzaville et le maire de Brazzaville; les commissaires de police d'arrondissements et les maires d'arrondissements ${ }^{22}$. La reconsidération de ces rapports a l'avantage, pour chaque service, d'éviter des incursions directes dans le pré carré des autres, ce qui éviterait la confusion et le chaos dans l'administration de l'espace public.

Notons qu'à Brazzaville, l'administration de l'espace public s'inscrit dans une dynamique conflictuelle au sens de Jean Bauchard (1981). Celui-ci considère qu'à l'origine d'un conflit il existe un antagonisme directeur qui se traduit par un ou plusieurs foyers susceptibles d'induire des champs de 
bataille où l'on peut mettre en lumière des convergences et des divergences d'intérêts entre acteurs. Cette dynamique conflictuelle peut se résumer ainsi qu'il suit :

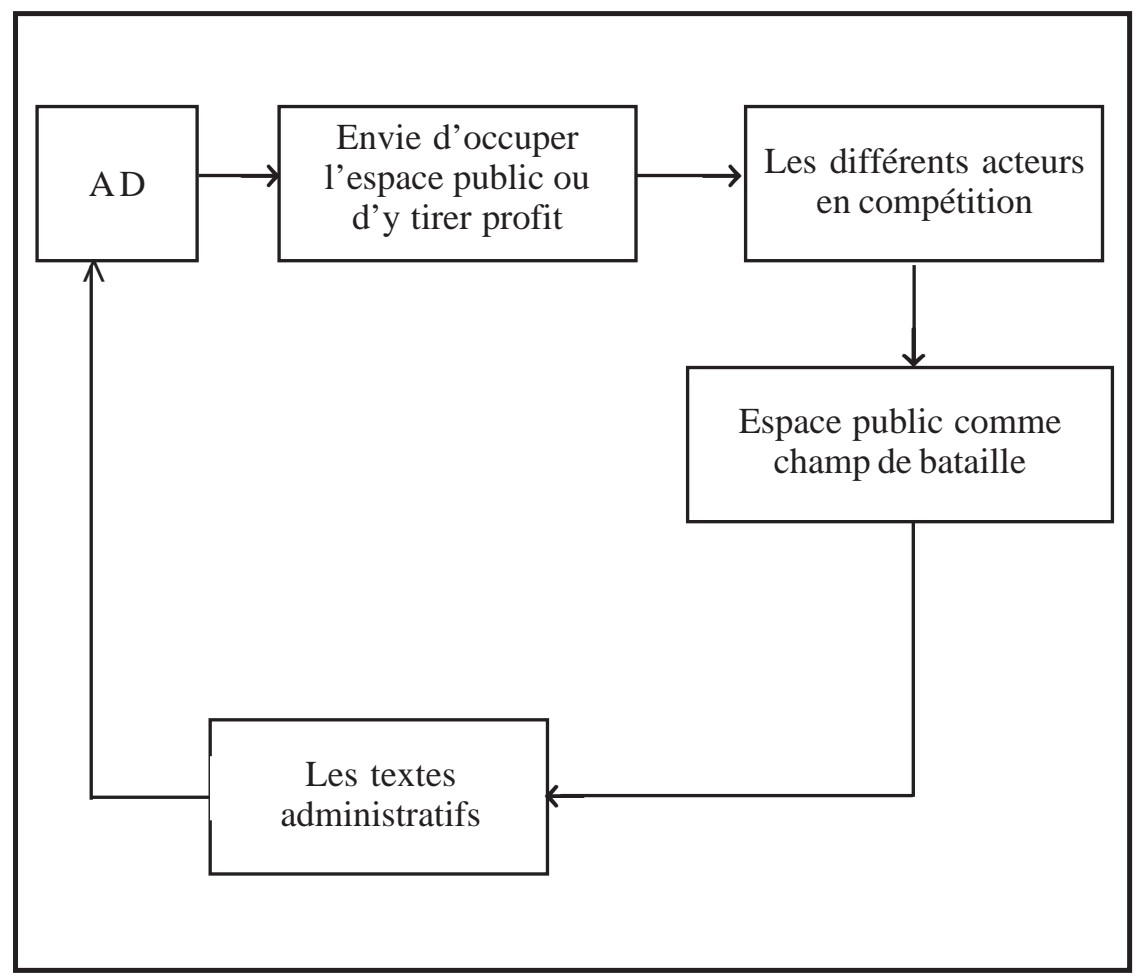

Il ressort de cette dynamique conflictuelle l'apparition des champs de bataille (réels ou potentiels) traduisant des alliances et des conflits, compte tenu des rapports de force dans le jeu des acteurs que sont : les propriétaires fonciers, la mairie centrale, les arrondissements, la police, les occupants de l'espace, etc.

\section{Scénarios de l'espace public à Brazzaville à l'horizon 2025}

Un scénario est à la fois une image du futur et un cheminement pour y parvenir (Moyo Nzololo 2008:9). L'avenir de l'espace public est envisagé à travers des futurs vraisemblables, c'est-à-dire par une probabilité non nulle de voir la réalisation du schéma modernisateur de la ville de Brazzaville par les pouvoirs publics. En octobre 2007, en effet, ils avaient annoncé médiatiquement et solennellement l'intégration de cette ville capitale dans la sphère des villes modernes du troisième millénaire. Dans ce scénario prospectif, l’horizon envisagé est 2025. 
Tenant compte de la vitrine spatiale actuelle, il y a lieu de construire deux scénarios (scénario chaos et scénario espoir), avec un contenu qui contraste. Ce contenu repose sur deux attitudes opposées face à l'avenir : une attitude d'agression grégaire de l'espace public par les citadins et une attitude de prise de conscience par les pouvoirs publics dans la réharmonisation et de la réarticulation de l'espace public.

Le "scénario chaos » révèle un avenir hypothéqué de l'espace public dans la mesure où le jeu des acteurs en place se polarise dans la matrice des luttes pour les intérêts personnels. Ces intérêts sont construits à partir des réseaux dynamiques, mais flous, entre administrateurs urbains et certains individus. En outre, ces intérêts induisent l'espace public urbain dans la dynamique conflictuelle. Ces conflits pourraient perdurer tant que l'espace public sera appréhendé par ses usagers comme un espace sûr d'expression et de lutte contre la pauvreté, les discriminations, la marginalisation et la paupérisation factorielle de plus en plus visible. Dans ce scénario, il y a aussi compromission de confiance entre notamment les services municipaux, la force publique et les usagers de l'espace public. Par exemple, la position des maraîchers des quartiers nord de Brazzaville demeure inflexible, car, pensentils, leurs intérêts sont menacés. Il y a en définitive une crise de confiance qui s'est installée depuis des décennies entre ces différentes entités. En rapport avec d'autres facteurs endogènes, cette crise peut être récurrente et permanente.

A côté de cette amalgame de pressions dont est victime l'espace public, il y a lieu de souligner l'enjeu environnemental. En effet, depuis quelques années, on assiste à de graves érosions dans la majorité des quartiers nord de Brazzaville. Ces érosions menacent dangereusement la sécurité sociale des populations habitant lesdits quartiers. Une des conséquences majeures de ces érosions est la progression massive et spectaculaire de la population vers l'espace public destiné à une utilité publique.

En revanche, le «scénario espoir » considère que l'avenir de l'espace public est certain, prometteur et rassurant. Cette assurance est à inscrire aux calendes et dans les dédales politiques actuels, dans la redéfinition d'une nouvelle image à imprimer à la ville d'ici 2025. Or l'amélioration de l'espace public est un passage obligé pour le revêtement de la ville des «tissus modernes ", authentiques.

Dans la vision prospective des pouvoirs publics, une tendance nette de reconsidération de l'espace public semble se dessiner. En redéfinissant et en distinguant les biens publics des biens privés, les pouvoirs publics cherchent à délimiter, à limiter et à éliminer la marge de manœuvre des prédateurs de l'espace dans les jours avenirs.

Le "scénario espoir » permet aussi d'inscrire l'espace public de Brazzaville dans une dynamique de transformation. Celle-ci se fait à partir 
d'un ensemble de mesures tendant vers la non aliénation de l'espace, par une indemnisation orientée de certains occupants de l'espace. La finalité de cette indemnisation vise le retour effectif de certains espaces publics à l'autorité de l'Etat. A l'analyse, les autorités congolaises envisagent le développement urbain durable qui induit des politiques et des stratégies urbaines globales, intégrant le social, l'environnement, le culturel et l'économique. Concernant l'environnement par exemple, et afin de faire face aux défis qu'il lance à l'ensemble de la population, les pouvoirs publics s'emploient à réaménager l'espace des quartiers nord menacés par les érosions. Et il semblerait que le succès de cette opération de grande envergure passe par une délocalisation temporaire des populations habitant les quartiers mis à l'épreuve par l'environnement. Pour ce faire, plus de 9000 citoyens seront déplacés par les pouvoirs publics dans le cadre de la modernisation de ces quartiers.

\section{Conclusion}

Peu de mots suffisent pour conclure cette réflexion. L'espace public de Brazzaville est un champ de compétition ouverte aux différentes catégories sociales. A des taux divers, ces couches sociales aliènent l'espace public qu'ils croient être un bien populaire sans maître, sans pouvoir, sans autorité publique. L'analyse des différentes pratiques qui s’y réalisent révèle que la question de la pauvreté est devenue le référent crucial qui pousse les gens en masse vers l'espace public où ils croient, enfin, trouver les solutions appropriées aux différents défis qui se présentent à eux.

L'espace public en tant que bien public est dans une insécurité croissante dont les pouvoirs publics alimentent les enjeux. Tenant compte du comportement populaire dans les rapports qu'il engendre systématiquement avec l'espace, les dysfonctionnements administratifs épinglés tout au long de cette étude sont de nature à soustraire la ville des fonctions traditionnelles qui lui sont dévolues. Il est donc important que l'espace public soit doté d'une politique d'administration sûre, avec une structure de commandement bien connue et bien organisée. Les textes administratifs sur l'espace public existent, et ils sont initiés progressivement. C'est bien. Mais ils sont d'excellentes fractures, parce qu'ils sont interprétés et appliqués différemment par les garants de l'espace urbain. Ceux-ci n'établissent point un lien, une «tri-unité » logique entre leurs concepteurs, l'espace public et leurs prédateurs.

Tout en cherchant à conserver ou à gagner individuellement les profits qui sont associés à l'espace public, certaines autorités administratives cherchent à contourner simultanément - volontairement - les exigences liées à la « consommation privée» de l'espace public. En soulignant cette 
complicité, il faut rappeler le désengagement de l'Etat, hier maître de la providence, à assurer le bien-être collectif. A l'analyse, le laisser-faire sur l'espace public constitue, pour d'autres autorités politico-administratives, un moyen efficace d'atténuer ou de stopper net la colère des masses indociles face à un ordre (pouvoir) défaillant.

\section{Notes}

1. A cet effet, lire avec intérêt Florence Bernault dans son ouvrage Démocraties ambiguës en Afrique centrale, Paris, Karthala, 1996, pp. 285-286.

2. Document anonyme sur Brazzaville, Ministère du Plan.

3. République Populaire du Congo : Commune de Ouenzé. Esquisse des données générales sur l'arrondissement 5 Ouenzé, 1983 (document d'archives).

4. L'exemple du quartier Simba Pelle.

5. Dans les quartiers où on trouve ces espaces publics, il se pose de graves problèmes d'électricité, de transport, de lotissement, d'assainissement et d'adduction d'eau potable.

6. Le transport dans ce secteur est devenu plus intense au lendemain de la guerre du 5 juin 1997.

7. Délibération $n^{\circ} 6$ du 11 février 2004.

8. Ce constat transparaît dans plusieurs documents administratifs consultés à l'Hôtel de Ville de Brazzaville.

9. Au sujet des crises foncières concernant la ceinture maraîchère de Brazzaville, on peut par exemple lire Robert Edmond Ziavoula dans « Les scènes foncières de Brazzaville » in Brazzaville, une ville à reconstruire, Paris, Karthala, 2006, pp. $246-249$.

10. Le 1er septembre 2008, plus de 100 maraîchers et propriétaires fonciers ont assiégé le Ministère de l'agriculture, en signe de protestation contre les décisions de ce ministère contre eux, alors que, selon la Primature, la situation concernant la ceinture maraîchère de Mpila et Talangaï est sur la table du Gouvernement et l'enveloppe prévue pour exproprier les maraîchers et les propriétaires fonciers est de 4 milliards de francs CFA. En réalité, pour des raisons des travaux à exécuter sur le site maraîcher évoqué dans le cadre de la municipalisation accélérée, le Ministère de l'agriculture propose que d'autres endroits (environ 4) soient légués aux maraîchers pour la suite de leurs activités maraîchères, ce que refusent les maraîchers qui ne veulent pas céder, pour expropriation, les surfaces qu'ils cultivent.

11. En 1986, Bernard Tchibambelela mentionnait déjà que l'occupation anarchique des terrains pour habitat de la ceinture maraîchère entraînait la disparition progressive de celle-ci, avec une perte de terrain de 60\%. « Problématique et choc de l'urbanisation au Congo : rapport avec l'alimentation » in La revue des Sciences Sociales n5, République Populaire du Congo, éditée par le Département de l'Idéologie et de la Formation Politique du Secrétariat du Comité Central du Parti Congolais du Travail, 1986, p.111. 
12. Dans l'imaginaire des Congolais, les Karachikas ont été injectés dans la sphère politique, avec les enjeux électoraux de 2002 et 2008 où est apparu un phénomène identique à celui de Karachika: les kata kata ou Sap-Sap. Ces derniers seraient des voleurs de sexe, sacrifiés ensuite de façon magique en vue de la conquête de l'électorat. Kata kata est de l'anthropologie linguistique lingala « couper ». Sap-Sap, quant à lui, est un système de transfert de crédit d'un numéro vers un autre. Il a été lancé par Zaim ex-Celtel Congo. Les sexes seraient pris par mutilation ou par simple disparition après un simple frottement entre individus.

13. La Nouvelle Espérance est le programme (projet de société) de compagne du président Dénis Sassou Nguesso. Il compte principalement quelque 14 points consécutifs. C'est sur la base de ce programme qu'il a été élu aux élections présidentielles de 2002.

14. Pour le compte de cette année 2008, 170 projets ont été sélectionnés.

15. Entre autres taux sanctionnant les infractions il y a : construction sans autorisation, $50.000 \mathrm{f}$ à $10.000 \mathrm{f}$ pour le Centre ville, $25.000 \mathrm{f}$ à $50.000 \mathrm{f}$ pour les quartiers populaires ; occupation anarchique du domaine public, $15.000 \mathrm{f}$ à $25.000 \mathrm{f}$ pour le centre-ville, $6.000 \mathrm{f} 12.000 \mathrm{f}$ pour les quartiers populaires ; défaut d'autorisation d'occuper une terrasse, $25.000 \mathrm{f}$ pour le centre-ville et $15.000 \mathrm{f}$ pour les quartiers populaires ; vente ambulante, $25.000 \mathrm{f}$ pour le centre-ville et $6.000 \mathrm{f}$ pour les quartiers populaires ; implantation des panneaux publicitaires sans autorisation, $25.000 \mathrm{f}$ à $50.000 \mathrm{f}$ pour le centre-ville et 15.000 $\mathrm{f}$ à $25.000 \mathrm{f}$ pour les quartiers populaires.

16. Dans le Document relatif à l'ordre public et la salubrité dans la ville de Brazzaville, conformément à la réunion de concertation du 24 avril 2003, le Colonel Jacques Antoine BOUITI faisait observer plusieurs points ayant un rapport étroit avec l'espace et le domaine publics. Il attirait l'attention des autorités municipales sur l'occupation anarchique des espaces publics par les organisateurs des cérémonies religieuses et autres.

17. Nous l'avons déjà signalé plus haut quand nous avons parlé des « Parkings réservés ".

18. Selon l'article 2 de la Délibération nº 06 du 11 février 2004, les taxes d'occupation de l'espace et du domaine publics se présentent comme suit : aire d'exposition des meubles, $20.000 \mathrm{f} / \mathrm{m}$ linéaire le trimestre ; occupation d'une portion de rue pour cérémonie, 5000f/24heures ; terrasse et barrestaurant centre-ville, 5000f/m2mois ; station lavage automobile, 3000f/m linéaire ; devanture d'un établissement centre-ville, 5000f/m2/mois ; devanture d'un établissement quartier, 3500f/m2/mois ; dépôt de matériaux de construction, 2000f/24heures ; construction en matériaux précaires ou amovibles, $4500 \mathrm{f} / \mathrm{m} 2 /$ mois.

19. Leur pouvoir est devenu plus inquiétant avec la dissolution des conseils d'arrondissements par l'acte fondamental. 
20. C'est le cas par exemple des occupants d'espace situé dans les environs du marché Plateau des 15 ans qui détiennent des titres délivrés par le maire de Moungali pendant la transition de 1992, alors qu'il existe un parking aménagé par la mairie centrale et destiné à l’usage collectif.

21. Pour la même nature de la demande, les prix varient parfois d'un arrondissement à un autre.

22. Le Colonel Jacques Antoine Bouiti a visiblement manifesté ce souhait dans le « Document relatif à l'ordre public et la salubrité dans la ville de Brazzaville, conformément à la réunion de concertation du 24 avril 2003 ».

\section{Bibliographie}

Abdou, Maliq, S., 1998, Mutations urbaines en Afrique. Document de travail 3/97, Dakar, CODESRIA.

Ayessa, J.J., 1999, Gestion de l'occupation du domaine public municipal : cas de la Commune de Brazzaville, Mémoire pour l'obtention du diplôme de l'ENAM, Université Marien Ngouabi, Brazzaville.

Bauchard, J., 1981, La dynamique conflictuelle, Paris, Editions Réseaux.

Bernault, F., 1996, Démocraties ambiguës en Afrique centrale, Paris, Karthala, 1996.

Féindangai, B., 2004, « Pratiques et représentations de l'espace chez les habitants des Kodros de Bangui ", in Rupture-Solidarité. Rites et possessions, № 5, Paris, Karthala, pp.239-250.

Moyo, N., 2008, Les transports en commun à Brazzaville à l'horizon 2020. Analyse prospective, Pointe-Noire, Editions ICES.

Tchibambelela, B., 1986, « Problématique et choc de l'urbanisation au Congo : rapport avec l'alimentation ", in La Revue des Sciences Sociales $n^{\circ} 5$, République Populaire du Congo, éditée par le département de l’Idéologie et de la Formation Politique du Secrétariat du Comité Central du Parti Congolais du Travail, 1986, pp. $101-113$.

Vennetier, P., 1985, « De la théorie à la pratique : code foncier et lotissements périurbain en R. P. du Congo », in Nicole Haumont et Alain Marie, (dir.), Politiques et pratiques urbaines dans les pays en voie de développement, tome 1, Paris, L'Harmattan, pp. 1998-211.

Ziavoula, R.E., 1988, " La course à l'espace urbain : les conflits fonciers à Brazzaville », in Politique Africaine, $n^{\circ} 31$. Le Congo, banlieue de Brazzaville, Paris, Karthala, pp.22 - 29.

Ziavoula, R.E., 2006, « Les scènes foncières de Brazzaville », in Brazzaville, une ville à reconstruire, Paris, Karthala, pp. 246 - 249. 